\title{
Scaling up the Anderson transition in random-regular graphs
}

\author{
M. Pino \\ Institute of Fundamental Physics IFF-CSIC, Calle Serrano 113b, Madrid 28006, Spain
}

(Received 8 September 2020; revised 14 October 2020; accepted 22 October 2020; published 20 November 2020)

\begin{abstract}
We study the Anderson transition in lattices with the connectivity of a random-regular graph. Our results show that fractal dimensions are continuous across the transition, but a discontinuity occurs in their derivatives, implying the existence of a nonergodic metallic phase with multifractal eigenstates. The scaling analysis gives critical exponent $v=0.94 \pm 0.08$ and critical disorder $W_{\mathrm{c}}=18.17 \pm 0.02$. Our data support that ergodicity is only recovered at zero disorder.
\end{abstract}

DOI: 10.1103/PhysRevResearch.2.042031

\section{INTRODUCTION}

Anderson localization of a single particle is crucial to understand transport in disordered materials [1]. Interactions between localized states also have important effects on those materials as, for instance, they affect the phononassisted conduction at low temperature [2-6]. Fleishman and Anderson argued that the Anderson transition, thus localization, survives upon including short-range interactions in three dimensions [7]. Altshuler and co-workers later found a metal-insulator transition, the many-body localization one, for disordered and interacting one-dimensional systems where all single-particle states are localized [8]. Besides the field of low-temperature transport, localized many-body states are playing a key role in several research areas such as that of quantum computing [9-11].

The degree of ergodicity of the metal near the many-body localization transition is not clear yet. An ergodic wave function roughly means that it has a uniform amplitude in the region of Hilbert space allowed by symmetry constraints [12]. Some groups have observed that metallic wave functions near this transition are not ergodic [13-18], while others support their ergodicity [19-22] and critical level statistics reported near the transition [23]. Given this controversy, it is sensible to step down and analyze simpler models that may exhibit similar behavior to that expected in the metallic side of the many-body localization transition.

Two relatively simple Hamiltonians are already known to exhibit a phase of nonergodic states. The first one corresponds to a particle hopping in a disordered Bethe lattice [25,26], a quite relevant model due to its ability to describe the decay of a local excitation in many-body disordered systems [27,28]. The second one is the Rosenzweig-Porter model of random matrices [29,30], whose nonergodic extended phase has

Published by the American Physical Society under the terms of the Creative Commons Attribution 4.0 International license. Further distribution of this work must maintain attribution to the author(s) and the published article's title, journal citation, and DOI. recently been characterized in Ref. [31] followed by many other studies [32-40]. Both models have a critical exponent $v=1$ at the Anderson transition, while the RosenzweigPorter one exhibit an additional nonergodic to ergodic transition with $v=1$ [41].

The next relatively simple Hamiltonian that may contain a phase of nonergodic metallic states is the one analyzed here, which describes a particle hopping in a random-regular graph. This graph is locally equivalent to a Bethe lattice but has closed loops with lengths that scale with the total number of nodes (see Fig. 1). Several studies predicted a nonergodic metallic regime in a random-regular graph with branching number $k=2$ [24,25,42], while recent ones have supported the ergodicity of the metal in general random graphs [43-45]. In Refs. [44,46,47], it is argued that nonergodic behavior is caused by large finite-size effects due to a correlation volume that diverges exponentially at the metallic side of the transition with critical exponent $v_{m}=1 / 2$, which is different from the critical exponent in the localized side $v_{l}=1$ [48]. In summary, the previous bibliography contains contradictory results regarding the ergodicity of the metallic side of random graphs, although recent studies point to its ergodicity [43-47].

Here, we present results that strongly support the nonergodicity of the metal in random-regular graphs with branching number $k=2$. We have performed a finite-size analysis for the first two and infinite moments of the wave-function amplitudes [49] [see Fig. 1(c)]. Using the scaling hypothesis [50,51], we are able to determine the critical disorder $W_{\mathrm{c}}=18.17 \pm 0.02$ and the correlation length critical exponent $v=0.94 \pm 0.08$ at the Anderson transition, this value is compatible with the theoretical prediction in Ref. [25] and similar to the one in the Bethe lattice and Rosenzweig-Porter random matrices [41].

\section{MODEL}

The Hamiltonian for a particle hopping in a random-regular graph is $(\hbar=1)$

$$
H=\sum_{i=1}^{N} \phi_{i} c_{i}^{\dagger} c_{i}+\sum_{\langle i j\rangle} c_{i}^{\dagger} c_{j}+c_{j}^{\dagger} c_{i},
$$


(a) Bethe lattice

(b) Random-regular graph
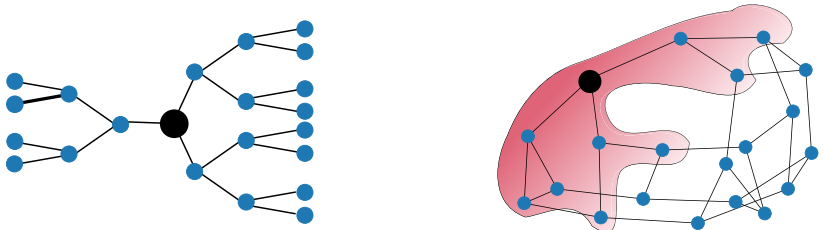

(c) Fractal dimensions in a random-regular graph

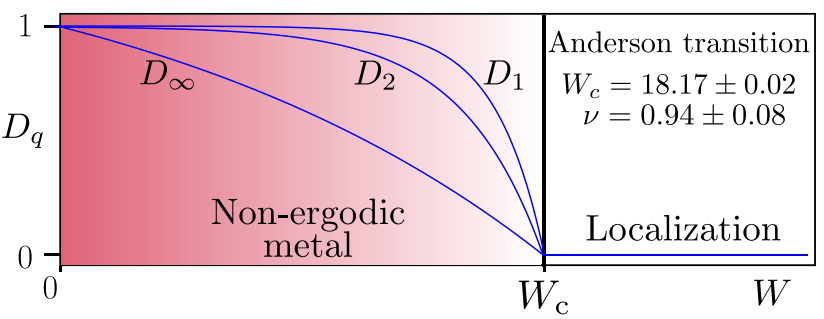

FIG. 1. A Bethe lattice (a) is similar to the local structure of a random-regular graph (b). A particle localized in the shaded area around the wide node (b) sees a graph analogous to that of a Bethe lattice (a). (c) Sketch of fractal dimensions for the Anderson model in a random-regular graph. The nonanalytical behavior at the Anderson transition is due to a discontinuity in the derivative of the fractal dimensions $D_{q}$, but fractal dimensions are continuous, implying the nonergodicity of the metal. Our finite-size results indicate that no additional phase transition exists from nonergodic to ergodic metals, contrary to the findings in Ref. [24]. Using the scaling hypothesis, we obtain the critical parameters shown in the figure.

where $c_{i}, c_{i}^{\dagger}$ are fermionic destruction and creation operators. A realization of this model implies choosing on-site random potentials; we use a box distribution for $\phi_{i}$ in $\left[-\frac{W}{2}, \frac{W}{2}\right]$, and a random-regular graph with branching number $k=2$. The graph has $N$ vertices (or sites) and the second summation in the Hamiltonian runs over its edges. We use the NETWORKX python package to generate the graphs [52].

We compute only one eigenstate at the center of the band for each realization of the previous Hamiltonian using SLEPC for Python [53-57]. We note that averaging with several eigenstates for the same Hamiltonian may induce correlations which undermine the precision of the scaling analysis [58]. The average over different realizations of the Hamiltonian is denoted by $\langle\cdots\rangle$ and around 50000 samples were used to average the largest size $N=128000$.

We will extrapolate data for finite graphs in order to obtain properties in the thermodynamical limit $N \rightarrow \infty$. The variable that controls finite-size effects in the finite-dimensional Anderson model is the length $L=N^{1 / d}$, where $d$ is the dimension. In other words, finite-size effects are controlled by the radius $L$ of the smallest hypersphere that contains all the nodes of the system. In a random-regular graph, we can define a "ball" of radius $L$ as containing all the points which appear after $L$ generations from the center. The number of nodes in such a ball scales as $N \sim k^{L}$, so we can use $L=\log (N)$ as the quantity that controls finite-size effects in random graphs.

\section{MULTIFRACTAL DIMENSIONS}

We characterize how uniform the wave-functions amplitudes are using the fractal dimensions $D_{q}=\log _{N}\left(I_{q}\right) /(1-q)$, where the moments are defined as $I_{q}=\left\langle\sum_{i=1}^{N}\left|\psi_{i}\right|^{2 q}\right\rangle$ and $\left|\psi_{i}\right|^{2}$ is the wave-function amplitude at site $i$ (see Supplemental Material [59] for a brief introduction to multifractal analysis). We are interested in the first two critical dimensions $D_{1}, D_{2}$ and in $D_{q \rightarrow \infty}$. The first one, $D_{1}$, can be computed from the participation entropy $S=\left\langle\sum_{i}\left|\psi_{i}\right|^{2} \log \left|\psi_{i}\right|^{2}\right\rangle$, as $S=-D_{1} \log (N)+c$. The last one is computed from the maximum wave-function amplitude $D_{\infty}=-\log _{N}\left(\max \left|\psi_{i}\right|^{2}\right)$, as introduced in Ref. [60]. We note that fractal dimensions are monotonous decreasing functions of $q$, that is, $\frac{d}{d q} D_{q} \leqslant 0$ (see Supplemental Material).

The fractal dimensions for an Anderson localized wave function are $D_{q}=0$ for $q \geqslant 1$, as those wave functions have a finite support set. Indeed, the number of sites $N_{\xi}$ with a finite wave-function amplitude does not scale with lattice size $N$ but it can be approximated by a geometric series $N_{\xi} \approx$ $k^{\xi+1}-1$, being $\xi$ the localization length. On the other hand, the support set of a metallic eigenfunction scales as a power of the total system size $N^{D}$ with $0<D \leqslant 1$ (it can be shown that $\left.D=D_{1}[25,42]\right)$. An ergodic state described by a Gaussian ensemble has vanishingly small wave-function-amplitude fluctuations around its average value so that $D_{q}=1$ for all $q$.

We need to determine whether the nonanalyticity at the Anderson transition is due to a discontinuity in the $D_{q}$ or in their derivatives with respect to disorder $D_{q}^{\prime}=\frac{d D_{q}}{d W}$. A direct transition from localized to ergodic states can only occur with a discontinuity in $D_{q}$ for $q \geqslant 1$, as in the threedimensional Anderson model [51]. On the other hand, there is a discontinuity in the derivative of fractal dimensions in the Rosenzweig-Porter model at the Anderson transition due to the multifractal nature of the metal in this model [41].

In Fig. 2, we have plotted $D_{q}^{\prime}$ computed with finite differences from the data for $D_{q}$ for $q=1,2, \infty$. The solid lines are the derivatives of Padé approximants for $D_{q}[60]$ (see the Supplemental Material [59] for more information about fractal dimensions and Padé approximants). Note that the derivatives of the Padé approximants agree quite well with the derivatives taken by finite differences. All the plots in Fig. 2 show a set of points where data for different sizes crosses around $W \approx 18$. The slope of $D_{q}^{\prime}$ at these crossing points gets steeper upon increasing system size. This is exactly the behavior expected in a phase transition with a discontinuity in the $D_{q}^{\prime}$, where the position of the crossing point marks the location of the critical point. We emphasize that no tendency to diverge is found for the fractal dimension derivatives around the expected critical disorder $W \approx 18$, which is strong evidence of the multifractal nature of the metal near the Anderson transition in randomregular graphs.

Our data for $D_{1}^{\prime}$ allows one to obtain an accurate determination of the critical disorder [see inset of Fig. 2(a)]. Indeed, there is a well-defined value of disorder $W$ where all curves cross, roughly at $W=18.17$. This estimation of the critical disorder is in excellent agreement with the theoretical result $W=18.17 \pm 0.01$ which was found in Ref. [44]. Crossing points can also be found for $D_{2}^{\prime}$ and $D_{\infty}^{\prime}$ around $W \approx 17$. Unlike data for $D_{1}^{\prime}$, those crossing points drift towards larger 

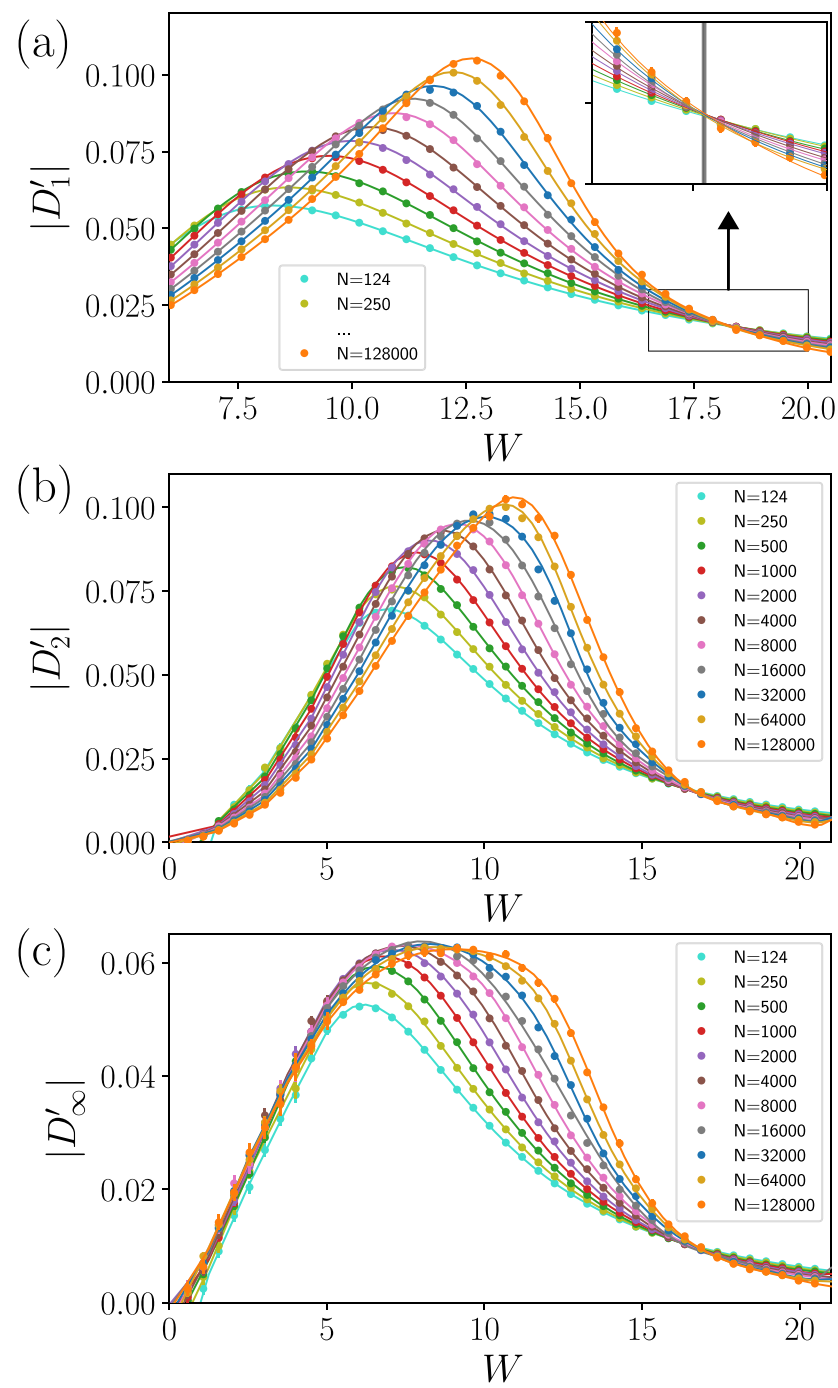

FIG. 2. Absolute value of the derivative of fractal dimensions with respect to disorder $D_{q}^{\prime}$ as a function of disorder $W$ for sizes $N=124,250, \ldots, 32000,64000,128000$. The solid lines are the derivative of Padé approximation for the fractal dimensions, respectively. The inset in (a) zooms in the critical region. The vertical line in this inset indicates our estimation of the critical point $W_{\mathrm{c}}=$ $18.17 \pm 0.02$.

values of the disorder, which indicates that finite-size corrections at criticality may be larger for those quantities than for $D_{1}^{\prime}$.

We can obtain additional information by looking at the maxima of fractal dimension derivatives as a function of system size. Note that the maximum for $\left|D_{\infty}^{\prime}\right|$ has already converged for the largest size in Fig. 2(c). We have checked that our finite-size data for the maximum of $\left|D_{1}^{\prime}\right|$ and $\left|D_{2}^{\prime}\right|$ are also compatible with a finite value in the thermodynamical limit (see Supplemental Material [59] for the extrapolation of these maxima). Thus, our numerical results do not support the divergence of fractal dimensions derivative at any value of disorder, which points again to the multifractal nature of the metal in random-regular graphs.
The results shown so far indicate that there is not an additional phase transition, different from the Anderson one, between nonergodic and ergodic metals. The case of a first -order transition, as predicted in Ref. [25], would imply a divergence of $D_{q}^{\prime}$ in the thermodynamic limit which can be ruled out as explained in previous paragraph. The possibility of a second-order transition from nonergodic to ergodic metal, with a discontinuity in $D_{q}^{\prime}$ as in the Rosenzweig-Porter model [41], is unlikely to occur. A second set of crossing points, different from the ones signaling the Anderson transition, are needed in order to have this additional phase transition. Although crossing points occur in all the fractal dimension derivatives at $W<14$, these are not compatible with a phase transition because the slope at the crossing remains roughly constant or decreases.

\section{SCALING AND CRITICAL PROPERTIES}

In the following we will use the scaling hypothesis to analyze the data for $D_{1}^{\prime}$ and extract critical parameters. We choose this quantity because it shows the smallest drift of the crossing points at the Anderson transition [see inset of Fig. 2(a)]. The fact that $D_{1}^{\prime}$ remains constant upon increasing system size at the crossings implies that irrelevant corrections are small.

Near a second-order phase transition, the singular part of a quantity can be expressed for a finite size $L$ as $[51,61,62]$

$$
A=L^{\zeta / v} f\left(\rho L^{1 / v}\right)
$$

where $f$ is the scaling function and $\rho=\left(W-W_{\mathrm{c}}\right)$ near the critical point, although expansion of higher orders may be needed when the analysis is performed for larger values of $\left|W-W_{c}\right|$. In order to scale $D_{1}^{\prime}$, we will replace $L$ by $\log (N)$ in Eq. (2) as discussed in Sec. II and set $\zeta=0$ as the value of $D_{1}^{\prime}$ remains constant at the transition. The scaling analysis consists in choosing $W_{\mathrm{c}}$ and $v$, so that all the curves for $D_{1}^{\prime}$ collapse when plotted as a function of the scaling variable $x=L^{1 / v}\left(W-W_{\mathrm{c}}\right)$ close enough to the critical point.

We have fitted the data of $D_{1}^{\prime}$ to the law in Eq. (2) for diffrent disorder intervals and expansion for $f$ and $\rho$ [Fig. 3(a)]. The best-quality fitting, the one with reduced chi-square closer to 1 , is obtained for an interval of disorder $[15,21]$ and orders in the field and scaling function $(0,5)$ [see Fig. 3(b)]. It gives $W_{\mathrm{c}}=18.17 \pm 0.02$ and critical exponent $v=0.90 \pm 0.01$. We rely on this estimation of the critical disorder as it is stable under changes in the fitting parameters [see Fig. 3(a)]. However, the estimation of $v$ is not stable under a change of the fittiing parameters. For instance, a fitting with expansions $(3,5)$ produces a reduced chi-square of 0.93 and an estimation of $v=0.95 \pm 0.04$ [file 5 of Fig. 3(a)]. We should be careful when discriminating between different fitting models with small differences in the reduced chi-squared. Indeed, the goodness-of-fit test using this statistic is less reliable for nonlinear models [63] and our estimation of error bars may not be fully accurate, as computing $D_{1}^{\prime}$ using finite difference introduces a correlation between different points.

We use the Padé approximants computed for $D_{1}$ and appearing as solid lines in Fig. 2(a), to obtain a better estimation of the critical exponent $v$. Applying the scaling ansatz Eq. (2) 
(a)

\begin{tabular}{cccccc}
\hline \hline$W$ & Order & $\chi_{r}^{2}$ & $\mathrm{np}$ & $W_{\mathrm{c}}$ & $\nu$ \\
\hline$[16,21]$ & $(0,3)$ & 2.4 & 81 & $18.18 \pm 0.02$ & $0.82 \pm 0.01$ \\
{$[16,21]$} & $(0,5)$ & 0.80 & 81 & $18.18 \pm 0.02$ & $0.91 \pm 0.02$ \\
{$[15,21]$} & $(0,3)$ & 5.7 & 99 & $18.13 \pm 0.02$ & $0.786 \pm 0.009$ \\
{$[15,21]$} & $(0,5)$ & 0.98 & 99 & $18.17 \pm 0.02$ & $0.90 \pm 0.01$ \\
{$[15,21]$} & $(3,5)$ & 0.93 & 99 & $18.14 \pm 0.03$ & $0.95 \pm 0.04$ \\
{$[14,21]$} & $(2,6)$ & 1.21 & 117 & $18.17 \pm 0.03$ & $0.95 \pm 0.02$ \\
{$[14,21]$} & $(3,6)$ & 1.17 & 117 & $18.16 \pm 0.03$ & $0.89 \pm 0.03$ \\
{$[15,18.1]$} & $(1,4)$ & 0.95 & 54 & $18.11 \pm 0.06$ & $0.84 \pm 0.09$ \\
\hline \hline
\end{tabular}

(b)

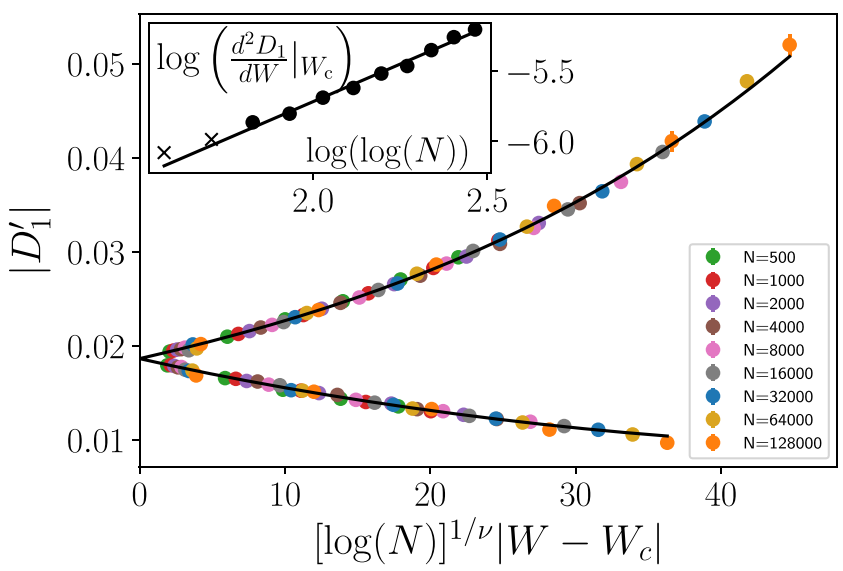

FIG. 3. Fitting the derivative of first fractal dimension $D_{1}^{\prime} \quad$ to a scaling form $A=f\left\{\left(\rho[\log (N)]^{1 / v}\right)\right\}$, for sizes $N=500,1000,2000, \ldots, 64000,128000$. (a) Results for fitting with $W$ disorder interval, order $(i, j)$ in the expansions $\left[\rho=\sum_{k=0}^{i} a_{k}\left(W-W_{\mathrm{c}}\right)^{k}\right.$ and $\left.f=\sum_{k=0}^{j} b_{k} x^{k}\right]$, reduced chi-square, $\chi_{r}^{2}$, and np, number of data points. The last two columns contain the results for the critical disorder and exponent $W_{\mathrm{c}}, v$ respectively. (b) Plot of $D_{1}^{\prime}$ data points and scaling function (solid line) as a function of the scaling variable $x=\left|W-W_{\mathrm{c}}\right|[\log (N)]^{1 / v}$ for $W=[15,21]$ and $(i, j)=(0,5)$. Those parameters produced the best quality fitting following the criteria of closest to 1 reduced-chi square. The result of the two coefficients in the scaling function for this best-quality fitting are $b_{0}=(18.7 \pm 0.1) \times 10^{-3}$ and $b_{1}=(3.5 \pm 0.1) \times 10^{-4}$. The inset of (b) contains the logarithm of the second derivative of the Padé approximants for $D_{1}$ with respect to disorder at $W_{\mathrm{c}}$ as a function of $\log [\log (N)]$. The solid line is a fit to a straight line of the points represented by $\bullet$ (the smallest sizes $x$ are excluded). It gives slope and origin $a=1.06 \pm 0.09$ and $b=-7.84 \pm 0.04$. The critical exponent $v=0.94 \pm 0.08$ is estimated as the inverse of the slope of the resulting straight line.

to $D_{1}^{\prime}=\frac{d D_{1}}{d W}$, we obtain

$$
\log \left(\left.\frac{d^{2} D_{1}}{d W}\right|_{W_{\mathrm{c}}}\right)=\frac{1}{v} \log [\log (N)]+\log \left[f^{\prime}\left(W_{\mathrm{c}}\right)\right],
$$

where $f^{\prime}$ is the derivative of the scaling function. In the inset of Fig. 3(b), we can see the left-hand side of Eq. (3) as a func- tion of $\log [\log (N)]$, where the derivatives have been computed analytically from the Padé approximants. The solid curve is a fit of the points to a straight line. From the fitting, we obtain a critical exponent $v=0.94 \pm 0.08$ and $f^{\prime}\left(W_{\mathrm{c}}\right) \approx 3.9 \times 10^{-4}$. The last estimation is similar to the one found via the scaling approach, coefficient $b_{1}$ reported in the caption of Fig. 3 . The estimated critical exponent is compatible with several of the ones found using the scaling hypothesis in Fig. 3(a). We regard this value as our estimation of the critical exponent $v=0.94 \pm 0.08$. We emphasize that the error bars for $v$ obtained using this approach are more reliable than those coming from the full scaling in the main panel of Fig. 3(b), as they are estimated from a linear fit.

\section{DISCUSSION}

We have shown that fractal dimensions $D_{1}, D_{2}$, and $D_{\infty}$ are continuous across the Anderson transition but their derivatives are discontinuous. This implies the existence of a phase where the system is metallic but nonergodic as eigenstates are multifractal $0<D_{q}<1$. This is different from the situation in the three-dimensional Anderson model, where the multifractality of the eigenstate is restricted just to the critical point. We have not found any evidence of an additional phase transition, one from nonergodic to ergodic metal, so the whole metal is nonergodic for random-regular graphs. Even in this situation, fractal dimensions may be too close to 1 at intermediate values of disorder so that multifractality is only appreciable near the critical region.

Applying the scaling hypothesis to $D_{1}^{\prime}$, we have estimated a critical exponent $v=0.94 \pm 0.08$ and a critical disorder $W_{\mathrm{c}}=18.17 \pm 0.02$. Our critical disorder agrees well with the one reported in Ref. [44]. The estimation of the critical exponent is compatible with the prediction $v=1$ in Ref. [25] but disagrees with the theory built in Refs. [43,44,64]. Those works point to an asymmetric scaling with $v=0.5$ and $v=1$ at the metallic and insulating sides of the Anderson transition, respectively.

Our scaling approach is the same as the one used for the Rosenzweig-Porter model in Ref. [41], but differs from the one in Refs. [46,47]. There, different critical exponents at the metallic and insulating sides of the transition were allowed due to the poor scaling of the data when employing the usual scaling form. Apart from the asymmetric scaling, there are other reasons which can produce a deviation from the scaling hypothesis such as a low expansion of the field $\rho$ in Eq. (2) or the existence of irrelevant scaling. We have experienced that low expansion in the relevant field produces a substantial underestimation of the critical exponent, especially when fitting data far from the critical point at the metallic side.

\section{ACKNOWLEDGMENTS}

I thank A. Rodríguez, P. Serna, and J. J. García-Ripoll for useful discussion and comments. Financial support by Fundación General CISC (Programa Comfuturo) is acknowledged. The numerical computations have been performed in the cluster Trueno of the CSIC. 
[1] P. W. Anderson, Absence of diffusion in certain random lattices, Phys. Rev. 109, 1492 (1958).

[2] A. Efros and B. I. Shklovskii, Coulomb gap and low temperature conductivity of disordered systems, J. Phys. C 8, L49 (1975).

[3] J.-J. Kim and H. J. Lee, Observation of a Nonmagnetic Hard Gap in Amorphous In/InO $\mathrm{I}_{x}$ Films in the Hopping Regime, Phys. Rev. Lett. 70, 2798 (1993).

[4] R. Rosenbaum, Crossover from Mott to Efros-Shklovskii variable-range-hopping conductivity in $\operatorname{In}_{x} \mathrm{O}_{y}$ films, Phys. Rev. B 44, 3599 (1991).

[5] M. Pollak, M. Ortuño, and A. Frydman, The Electron Glass (Cambridge University Press, Cambridge, UK, 2013).

[6] M. Pino, A. M. Somoza, and M. Ortuño, Quantum Coulomb gap in low dimensions, Phys. Rev. B 86, 094202 (2012).

[7] L. Fleishman and P. W. Anderson, Interactions and the Anderson transition, Phys. Rev. B 21, 2366 (1980).

[8] D. Basko, I. Aleiner, and B. Altshuler, English metal-insulator transition in a weakly interacting many-electron system with localized single-particle states, Ann. Phys. 321, 1126 (2006).

[9] P. Roushan, C. Neill, J. Tangpanitanon, V. Bastidas, A. Megrant, R. Barends, Y. Chen, Z. Chen, B. Chiaro, A. Dunsworth et al., Spectroscopic signatures of localization with interacting photons in superconducting qubits, Science 358, 1175 (2017).

[10] C. R. Laumann, R. Moessner, A. Scardicchio, and S. Sondhi, Quantum annealing: The fastest route to quantum computation? Eur. Phys. J.: Spec. Top. 224, 75 (2015).

[11] B. Altshuler, H. Krovi, and J. Roland, Anderson localization makes adiabatic quantum optimization fail, Proc. Natl. Acad. Sci. U. S. A. 107, 12446 (2010).

[12] By ergodic we mean a system that obeys the laws of statistical mechanics, which for infinite temperature and using the eigenstate thermalization hypothesis implies that its eigenstates are described by the Gaussian matrix ensemble compatible with the symmetries of the system.

[13] M. Pino, L. B. Ioffe, and B. L. Altshuler, Nonergodic metallic and insulating phases of Josephson junction chains, Proc. Natl. Acad. Sci. U. S. A. 113, 536 (2016).

[14] M. Pino, V. E. Kravtsov, B. L. Altshuler, and L. B. Ioffe, Multifractal metal in a disordered Josephson junctions array, Phys. Rev. B 96, 214205 (2017).

[15] E. J. Torres-Herrera and L. F. Santos, Extended nonergodic states in disordered many-body quantum systems, Ann. Phys. 529, 1600284 (2017).

[16] R. Berkovits, Signature of non-ergodicity in low-lying excitations of disordered many-particle systems, Ann. Phys. 529, 1700042 (2017).

[17] S. Roy, I. Khaymovich, A. Das, and R. Moessner, Multifractality without fine-tuning in a Floquet quasiperiodic chain, Sci. Post. Phys. 4, 025 (2018).

[18] L. Faoro, M. V. Feigel'man, and L. Ioffe, Non-ergodic extended phase of the quantum random energy model, Ann. Phys. 409, 167916 (2019).

[19] D. J. Luitz and Y. B. Lev, The ergodic side of the many-body localization transition, Ann. Phys. 529, 1600350 (2017).

[20] D. J. Luitz, N. Laflorencie, and F. Alet, Extended slow dynamical regime close to the many-body localization transition, Phys. Rev. B 93, 060201(R) (2016).
[21] D. J. Luitz, N. Laflorencie, and F. Alet, Many-body localization edge in the random-field Heisenberg chain, Phys. Rev. B 91, 081103(R) (2015).

[22] M. Žnidarič, A. Scardicchio, and V. K. Varma, Diffusive and Subdiffusive Spin Transport in the Ergodic Phase of a ManyBody Localizable System, Phys. Rev. Lett. 117, 040601 (2016).

[23] C. L. Bertrand and A. M. García-García, Anomalous Thouless energy and critical statistics on the metallic side of the manybody localization transition, Phys. Rev. B 94, 144201 (2016).

[24] B. L. Altshuler, E. Cuevas, L. B. Ioffe, and V. E. Kravtsov, Nonergodic Phases in Strongly Disordered Random Regular Graphs, Phys. Rev. Lett. 117, 156601 (2016).

[25] V. Kravtsov, B. Altshuler, and L. Ioffe, Non-ergodic delocalized phase in Anderson model on Bethe lattice and regular graph, Ann. Phys. 389, 148 (2018).

[26] G. Biroli and M. Tarzia, Delocalized glassy dynamics and many-body localization, Phys. Rev. B 96, 201114(R) (2017).

[27] B. L. Altshuler, Y. Gefen, A. Kamenev, and L. S. Levitov, Quasiparticle Lifetime in a Finite System: A Nonperturbative Approach, Phys. Rev. Lett. 78, 2803 (1997).

[28] G. Biroli and M. Tarzia, Anomalous dynamics on the ergodic side of the many-body localization transition and the glassy phase of directed polymers in random media, Phys. Rev. B 102, 064211 (2020).

[29] N. Rosenzweig and C. E. Porter, "Repulsion of Energy Levels" in Complex Atomic Spectra, Phys. Rev. 120, 1698 (1960).

[30] A. Altland, M. Janssen, and B. Shapiro, Perturbation theory for the Rosenzweig-Porter matrix model, Phys. Rev. E 56, 1471 (1997).

[31] V. E. Kravtsov, I. M. Khaymovich, E. Cuevas, and M. Amini, A random matrix model with localization and ergodic transitions, New J. Phys. 17, 122002 (2015).

[32] D. Facoetti, P. Vivo, and G. Biroli, From non-ergodic eigenvectors to local resolvent statistics and back: A random matrix perspective, Europhys. Lett. 115, 47003 (2016).

[33] C. Monthus, Multifractality of eigenstates in the delocalized non-ergodic phase of some random matrix models: WignerWeisskopf approach, J. Phys. A: Math. Theor. 50, 295101 (2017).

[34] K. Truong and A. Ossipov, Eigenvectors under a generic perturbation: Non-perturbative results from the random matrix approach, Europhys. Lett. 116, 37002 (2016).

[35] P. von Soosten and S. Warzel, Non-ergodic delocalization in the Rosenzweig-Porter model, Lett. Math. Phys. 109, 905 (2019).

[36] M. Amini, Spread of wave packets in disordered hierarchical lattices, Europhys. Lett. 117, 30003 (2017).

[37] S. Bera, G. De Tomasi, I. M. Khaymovich, and A. Scardicchio, Return probability for the Anderson model on the random regular graph, Phys. Rev. B 98, 134205 (2018).

[38] G. De Tomasi, M. Amini, S. Bera, I. Khaymovich, and V. Kravtsov, Survival probability in generalized RosenzweigPorter random matrix ensemble, SciPost Phys. 6, 14 (2019).

[39] V. Kravtsov, I. Khaymovich, B. Altshuler, and L. Ioffe, Localization transition on the random regular graph as an unstable tricritical point in a log-normal Rosenzweig-Porter random matrix ensemble, arXiv:2002.02979.

[40] M. Tarzia, Many-body localization transition in Hilbert space, Phys. Rev. B 102, 014208 (2020). 
[41] M. Pino, J. Tabanera, and P. Serna, From ergodic to non-ergodic chaos in Rosenzweig-Porter model, J. Phys. A: Math. Theor. 52, 475101 (2019).

[42] A. De Luca, B. L. Altshuler, V. E. Kravtsov, and A. Scardicchio, Anderson Localization on the Bethe Lattice: Nonergodicity of Extended States, Phys. Rev. Lett. 113, 046806 (2014).

[43] K. S. Tikhonov, A. D. Mirlin, and M. A. Skvortsov, Anderson localization on random regular graphs, Phys. Rev. B 94, 220203(R) (2016).

[44] K. S. Tikhonov and A. D. Mirlin, Critical behavior at the localization transition on random regular graphs, Phys. Rev. B 99, 214202 (2019).

[45] G. Biroli and M. Tarzia, Delocalization and ergodicity of the Anderson model on Bethe lattices, arXiv:1810.07545.

[46] I. Garcia-Mata, O. Giraud, B. Georgeot, J. Martin, R. Dubertrand, and G. Lemarié, Scaling Theory of the Anderson Transition in Random Graphs: Ergodicity and Universality, Phys. Rev. Lett. 118, 166801 (2017).

[47] I. García-Mata, J. Martin, R. Dubertrand, O. Giraud, B. Georgeot, and G. Lemarié, Two critical localization lengths in the Anderson transition on random graphs, Phys. Rev. Res. 2, 012020 (2020).

[48] E. Cuevas, V. Gasparian, and M. Ortuño, Anomalously Large Critical Regions in Power-Law Random Matrix Ensembles, Phys. Rev. Lett. 87, 056601 (2001).

[49] T. C. Halsey, M. H. Jensen, L. P. Kadanoff, I. Procaccia, and B. I. Shraiman, Fractal measures and their singularities: The characterization of strange sets, Phys. Rev. A 33, 1141 (1986).

[50] M. E. Fisher and M. N. Barber, Scaling tTheory for Finite-Size Effects in the Critical Region, Phys. Rev. Lett. 28, 1516 (1972).

[51] A. Rodriguez, L. J. Vasquez, K. Slevin, and R. A. Römer, Critical Parameters from a Generalized Multifractal Analysis at the Anderson Transition, Phys. Rev. Lett. 105, 046403 (2010).

[52] A. Hagberg, P. Swart, and D. S Chult, Exploring network structure, dynamics, and function using networkx, Technical report No. LA-UR-08-05495, No. LA-UR-08-5495, Los alamos national lab (LANL), los alamos, NM, United States, 2008.
[53] V. Hernandez, J. E. Roman, and V. Vidal, SLEPc: A scalable and flexible toolkit for the solution of eigenvalue problems, ACM Trans. Math. Software 31, 351 (2005).

[54] V. Hernandez, J. E. Roman, and V. Vidal, SLEPc: Scalable library for eigenvalue problem computations, Lect. Notes Comput. Sci. 2565, 377 (2003).

[55] J. E. Roman, C. Campos, E. Romero, and A. Tomas, SLEPc users manual, Technical Report No. DSIC-II/24/02 - Revision 3.13, D. Sistemes Informàtics i Computació, Universitat Politècnica de València, 2020.

[56] V. Hernández, J. E. Román, and A. Tomás, Parallel Arnoldi eigensolvers with enhanced scalability via global communications rearrangement, Parallel Comput. 33, 521 (2007).

[57] L. D. Dalcin, R. R. Paz, P. A. Kler, and A. Cosimo, Parallel distributed computing using Python, Adv. Water Resour. 34, 1124 (2011), new Computational Methods and Software Tools.

[58] A. Rodriguez, L. J. Vasquez, K. Slevin, and R. A. Römer, Multifractal finite-size scaling and universality at the Anderson transition, Phys. Rev. B 84, 134209 (2011).

[59] See Supplemental Material at http://link.aps.org/supplemental/ 10.1103/PhysRevResearch.2.042031 for a brief introduction to multifractal analysis, fractal dimensions in random-regular graphs and the extrapolation of the maxima attained by their derivatives.

[60] J. Lindinger, A. Buchleitner, and A. Rodríguez, Many-Body Multifractality throughout Bosonic Superfluid and Mott Insulator Phases, Phys. Rev. Lett. 122, 106603 (2019).

[61] J. Cardy, Scaling and renormalization in statistical physics (Cambridge University Press, Cambridge, UK, 1996), Vol. 5.

[62] K. Slevin and T. Ohtsuki, Corrections to Scaling at the Anderson Transition, Phys. Rev. Lett. 82, 382 (1999).

[63] R. Andrae, T. Schulze-Hartung, and P. Melchior, Dos and don'ts of reduced chi-squared, arXiv:1012.3754.

[64] A. D. Mirlin and Y. V. Fyodorov, Distribution of Local Densities of States, Order Parameter Function, and Critical Behavior Near the Anderson Transition, Phys. Rev. Lett. 72, 526 (1994). 